\title{
Enhanced radiosensitivity of NSCLC cells by transducer of erbB2.1 (TOB1) through modulation of the MAPK/ERK pathway
}

\author{
KE-KANG SUN ${ }^{1,2^{*}}$, NING ZHONG $^{1 *}$, YANG YANG $^{2}$, LIN ZHAO $^{2}$ and YANG JIAO ${ }^{2}$ \\ ${ }^{1}$ Department of Gastrointestinal Surgery Division of Thoracic Surgery, Kunshan First People's Hospital \\ Affiliated to Jiangsu University, Kunshan, Jiangsu 215300; ${ }^{2}$ Key Laboratory of Radiation Biology, \\ School of Radiation Medicine and Protection, Medical College of Soochow University, \\ Suzhou, Jiangsu 215123, P.R. China
}

Received December 27, 2012; Accepted February 26, 2013

DOI: 10.3892/or.2013.2403

\begin{abstract}
Transducer of erbB2.1 (TOB1) is a member of the B-cell translocation gene (BTG)/transducer of erbB2 (TOB) anti-proliferative protein family. Previous studies have demonstrated that overexpression of TOB1 significantly enhances the radiosensitivity of breast and cervical cancer cells. However, the potential mechanisms of TOB1 are still debated. In the present study, we evaluated the effects of infrared (IR) radiation on TOB1 expression in the human lung cancer cell lines NCI-H1975 and A549 via western blot analysis. NCI-H1975 cells were transfected with TOB1 recombinant plasmid, and A549 cells were treated with TOB1-small interfering RNA (siRNA) to establish gain-of-function and loss-of-function cell models. The effects of radiation and TOB1 overexpression and silencing on clonogenic survival, cell cycle distribution and DNA repair were assessed. Western blot analysis was performed to determine the related mechanisms. The expression levels of TOB1 were significantly induced by IR radiation. Overexpression of TOB1 abrogated radiation-induced G2/M arrest, reduced clonogenic cell survival and enhanced $\gamma$-H2AX foci in NCI-H1975 cells exposed to irradiation. TOB1-siRNA demonstrated opposite effects in A549 cells. TOB1 regulated the activation of mitogen-activated protein kinase (MAPK) and modulated the phosphorylation of p53 via activation of the MAPK/extracellular signal-regulated kinase (ERK) pathway. The findings suggest that TOB1 may be a novel molecular
\end{abstract}

Correspondence to: Dr Yang Jiao, Key Laboratory of Radiation Biology, School of Radiation Medicine and Protection, Medical College of Soochow University, Renai Road, Suzhou, Jiangsu 215123, P.R. China

E-mail: jiaoyang@suda.edu.cn

${ }^{*}$ Contributed equally

Key words: transducer of erbB2.1, non-small cell lung cancer, radiosensitivity, MAPK/ERK target of irradiation. TOB1 modulated the radiosensitivity of lung cancer cells via the MAPK/ERK signaling pathway.

\section{Introduction}

Lung cancer is the leading cause of cancer-related deaths worldwide, among which non-small cell lung cancer (NSCLC) accounts for more than $80 \%$ of cases (1). Radiotherapy is becoming increasingly significant since it has been shown to improve local control and reduce recurrence of NSCLC. However, the use of radiotherapy is also confronted with dilemmas due to intrinsic radioresistance.

Transducer of erbB2.1 (TOB1) was identified as a member of the B-cell translocation gene (BTG)/transducer of erbB2 (TOB) anti-proliferative protein family, which was discovered in 1996 (2). Accumulated evidence confirms that TOB1 is involved in the negative regulation of cell growth and functions as a tumor suppressor (3). In previous studies in 2007 from our laboratory, Jiao et al (4) demonstrated that pre-irradiation treatment with adenovirus-mediated TOB1 significantly increased the radiosensitivity of breast cancer cells. However, whether TOB1 radiosensitizes NSCLC cells to ionizing radiation and whether or not its underlying mechanisms are associated with epidermal growth factor receptor (EGFR)-dependent signaling pathways has not been thoroughly elucidated.

In the present study, TOB1-overexpressing and -suppressed NSCLC cells were used to determine the clonogenic growth after radiation in order to investigate whether TOB1 affects the radiosensitivity of lung cancer cells. Radiation-induced double-strand break (DSB) formation and cell cycle redistribution were also detected. Western blot analysis was used to investigate the underlying molecular signaling and to identify the precise mechanisms of TOB1 in NSCLC cell lines in response to radiation.

\section{Materials and methods}

Cell culture and reagents. The human NSCLC cell lines NCI-H1975 and A549 were purchased from the American Type Culture Collection (Manassas, VA, USA). Cells were 
maintained in Dulbecco's modified Eagle's medium (DMEM) supplemented with $10 \%$ fetal calf serum (FCS), L-glutamine (5 mmol/l), non-essential amino acids $(5 \mathrm{mmol} / \mathrm{l})$, penicillin $(100 \mathrm{U} / \mathrm{ml})$ and streptomycin $(100 \mathrm{U} / \mathrm{ml})$ (Invitrogen, Carlsbad, $\mathrm{CA}$, USA), at $37^{\circ} \mathrm{C}$ in a humidified atmosphere with $5 \%$ $\mathrm{CO}_{2}$. Gefitinib (Iressa ${ }^{\circledR}$ ) was purchased from AstraZeneca (Macclesfield, UK) and the MEK1/2 inhibitor U0126 was obtained from Cell Signaling Technology, Inc. (Beverly, MA, USA).

Ionizing radiation. A Siemens $6 \mathrm{MV}$ X-ray linear accelerator was used to deliver a single dose of IR radiation, at a dose rate of $200 \mathrm{cGy} / \mathrm{min}$ at room temperature.

Plasmids, small interfering RNAs (siRNAs) and transfection. The full-length human TOB1 cDNA was derived using polymerase chain reaction (PCR), using specific primers designed according to the TOB1 reference sequence from GenBank (NM_005749.2), and then cloned into the eukaryotic expression vector pcDNA3.0 (Invitrogen). Three siRNAs that target TOB1 mRNA and control (scrambled-sequence) siRNA were designed and synthesized by Invitrogen. The Lipofectamine-introduced plasmid and siRNA transfection were performed as previously described (5). The expression of TOB1 was determined by western blot analysis.

Clonogenic survival assay. The cells were plated at different cell densities and irradiated with $6 \mathrm{~Gy} \mathrm{X}$-rays $24 \mathrm{~h}$ later. After 12-14 days of incubation at $37^{\circ} \mathrm{C}$, the cells were stained with Giemsa. The number of colonies per dish was counted and the surviving fractions were calculated as the ratio of plating efficiencies for irradiated and unirradiated cells. Plating efficiency was defined as the colony number divided by the number of cells plated for the unirradiated controls. Experiments were conducted in triplicate and data are presented as the means \pm standard deviation (SD) from three independent experiments. All survival fractions were fitted into the linear quadratic model.

Cell cycle analysis. The cells were removed with trypsin and collected into centrifuge tubes together with the culture medium. The detailed methods for flowcytometric analysis have been previously described (6). The cell cycle distribution was calculated from 10,000 cells using ModFit LT software (Becton-Dickinson, San Jose, CA, USA) and FACSCalibur (Becton-Dickinson).

Immunofluorescence assay. The immunofluorescence detection of $\gamma$-H2AX foci was applied for the determination of residual DNA DSBs. Cells grown on coverslips (Fisher Scientific) were fixed in ice cold $4 \%$ paraformaldehyde for 30 min, blocked with $3 \%$ bovine serum albumin (BSA) in phosphate-buffered saline (PBS) and incubated with the antibody phospho-H2AX (ser139, dilution 1:500; Millipore) for $2 \mathrm{~h}$ at $4^{\circ} \mathrm{C}$. After washing with PBS, the secondary FITCconjugated antibody was added for $1 \mathrm{~h}$, and the slides were washed with PBS and mounted with mounting medium containing DAPI. The slides were mounted with fluorescent mounting medium (Dako, Germany). For each treatment condition, $\gamma-\mathrm{H} 2 \mathrm{AX}$ foci were determined in $\geq 50$ cells and the data are presented as the means \pm SD from three independent experiments.

Western blot analysis. Western blot analysis was performed as previously described (5). The following primary antibodies were used for immunoblotting: $\beta$-actin (C-4), TOB1 (E-1), EGFR (53A5), NF- $\mathrm{B}$ (P65A), cyclin B1 (D-11), Cdc2 (B-5) and the secondary antibodies horseradish peroxidase (HRP)-labeled goat anti-mouse (GAM-007) and goat anti-rabbit (SC-2004) IgG (dilution, 1:1,000; Santa Cruz Biotechnology, Inc., Santa Cruz, CA, USA), as well as phospho-extracellular signal-regulated kinase (ERK) 1/2 (T202/Y204), ERK1/2, phospho-p38 (T180/Y182), p38 and phospho-p53 (serine 15) (dilution, 1:1,000; Cell Signaling Technology, Inc.).

Statistical analysis. The data are presented as the means \pm SD. Statistical comparisons of the experimental results between the treated and control groups were made using the two-tailed Student's t-test. All statistical tests were performed using SPSS version 17.0. $\mathrm{P} \leq 0.05$ was considered to indicate a statistically significant result.

\section{Results}

Radiation-induced TOB1 expression in human lung cancer cell lines. IR radiation stimulated TOB1 expression in NCI-H1975 and A549 lung cancer cell lines. The expression levels of TOB1 following various doses $(0-10 \mathrm{~Gy})$ of IR radiation in the two lung cancer cell lines were determined by western blot and densitometric analyses. Radiation-induced TOB1 expression was measured $24 \mathrm{~h}$ after radiation. A significant increase in the TOB1 protein level was observed when a dose of $\geq 2$ Gy was used (Fig. 1A). For the time-response induction of TOB1, the expression was increased at the earliest time-point tested $(6 \mathrm{~h})$ and was elevated at $24 \mathrm{~h}$ following 6 Gy of radiation (Fig. 1B). Little or no change in the expression of $\beta$-actin was observed in all of the experiments.

TOB1 regulates the radiosensitivity of lung cancer cell lines. NCI-H1975 cells were transfected with TOB1 recombinant plasmid to establish stable cells. TOB1-siRNA was induced into A549 cells to determine whether downregulation of TOB1 expression enhances the radioresistance of lung cancer cells. The efficacy of TOB1 recombinant plasmid and siRNA was confirmed by western blot analysis. Clonogenic assay was performed to assess the cell survival after radiation. TOB1 overexpression was combined with radiation and was found to reduce the clonogenic growth of NCI-H1975 cells compared with irradiated control cells. On the contrary, the number of colonies in the mock-transfection group was not affected. TOB1-siRNA transfection was combined with radiation and was found to induce clonogenic growth of A549 cells compared with irradiated control cells (Fig. 2).

TOB1 regulates cell cycle redistribution of lung cancer cells after radiation. Most mammalian cells exhibit transient delays in the G1 and G2 phases of the cell cycle after radiation treatment allowing the cell to correct possible defects (6). In the present study, we identified the effects of TOB1 on lung cancer cell cycle progression. After irradiation with $6 \mathrm{~Gy} \mathrm{X}$-rays, all the 

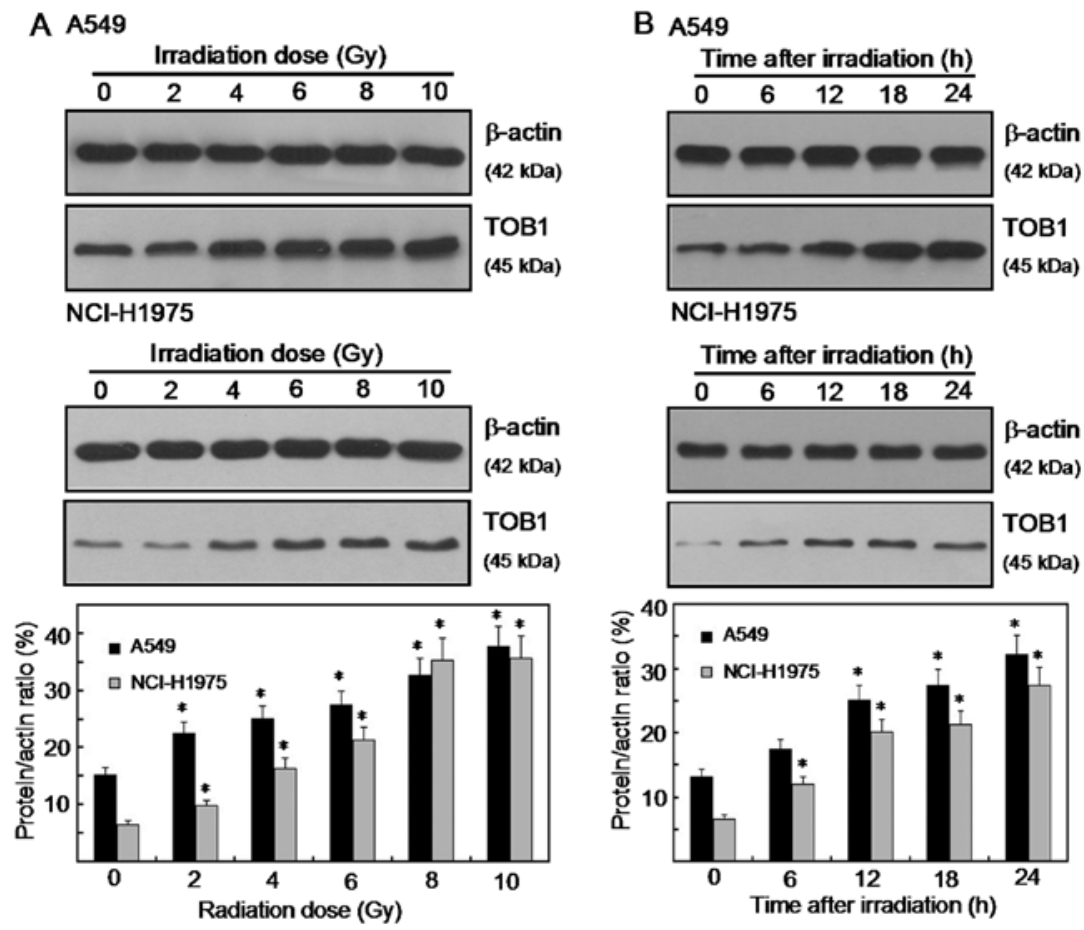

Figure 1. Irradiation upregulates TOB1 expression in the NSCLC cell lines A549 and NCI-H1975. (A) The cells were treated with various doses of irradiation and harvested for western blot analysis $24 \mathrm{~h}$ later. (B) The cells were treated with $6 \mathrm{~Gy}$ X-rays and harvested for protein analysis at different times. The densitometry values are the means of at least three independent experiments.
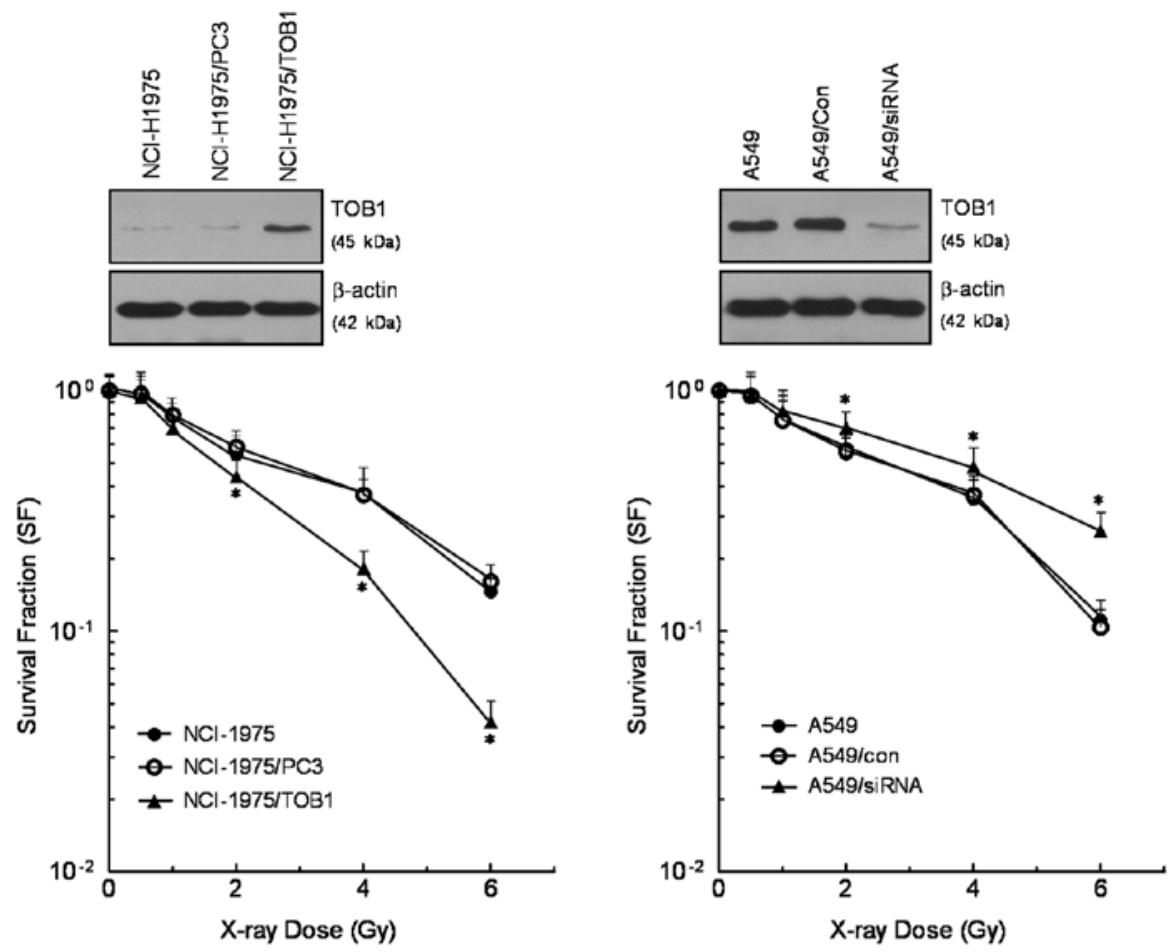

Figure 2. TOB1 regulates the radiosensitivity of lung cancer cell lines. The expression of TOB1 was confirmed by western blot analysis in the NCI-H1975/TOB1 transfectant and parental cells, as well as in the A549 and A549/siRNA cells. Cells transfected with the indicated plasmids or siRNA were irradiated with a single dose of $0.5,1,2,4$ and $6 \mathrm{~Gy}$. Unirradiated cells were used as control cells. Survival curves were obtained by means of standard colony formation assay. All the experiments were performed independently at least three times. ${ }^{*} \mathrm{P}<0.05$ vs. the control group.

above-described cells were analyzed for cell cycle distribution. As shown in Fig. 3A, radiation treatment significantly increased the percentage of G2/M phase cells. In NCI-H1975/TOB1 cells prior to radiation, the percentage of cells in the $\mathrm{G} 2 / \mathrm{M}$ phase was decreased compared with radiation alone $(t=7.75, \mathrm{P}<0.05)$. In A549/siRNA cells subjected to radiation, the percentage of 

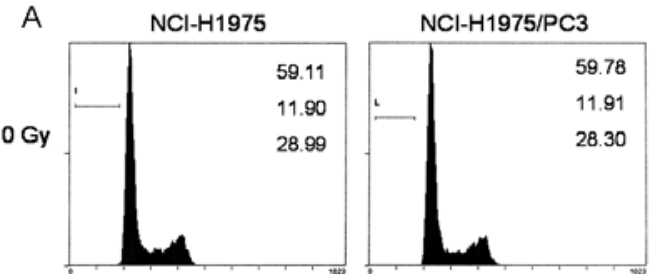

NCl-H1975/TOB1
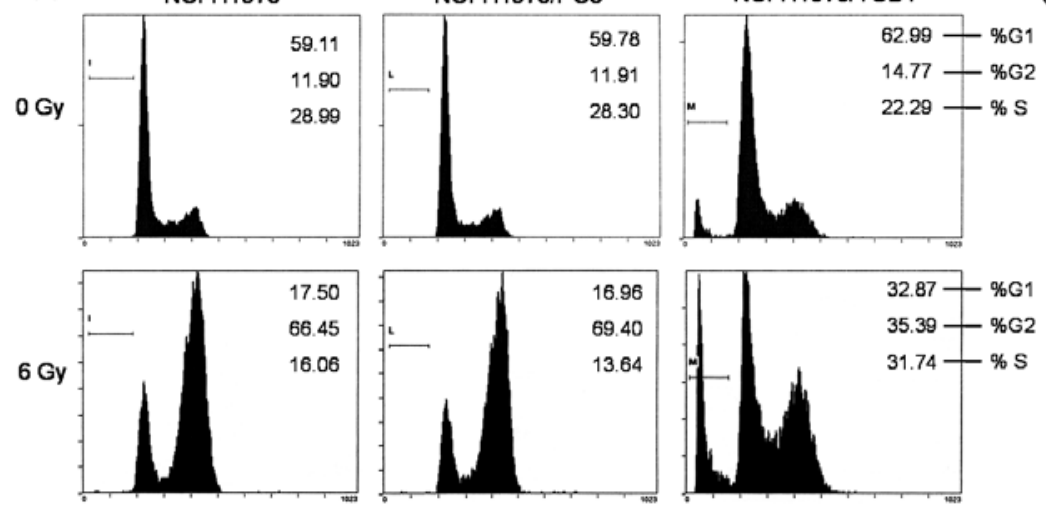

B

A549
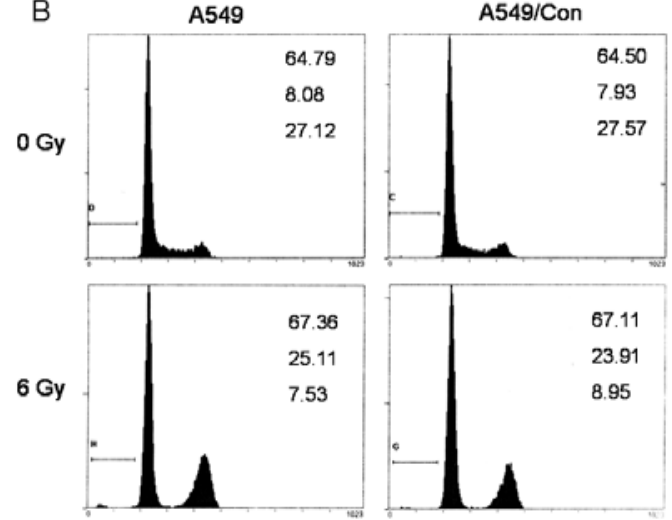

A549/siRNA

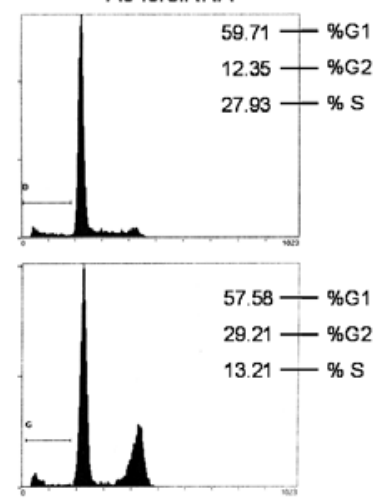

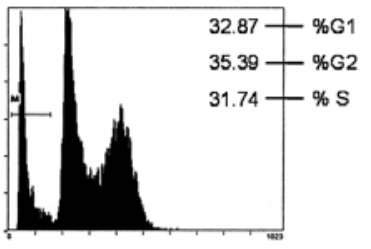

C

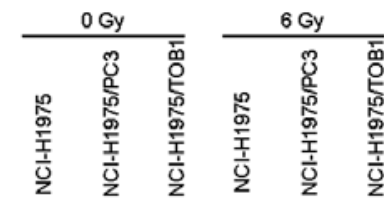

Cdc2

(34 kDa)

Cyclin B1

(55 kDa)

$\beta$-actin

$(42 \mathrm{kDa})$

\section{D}

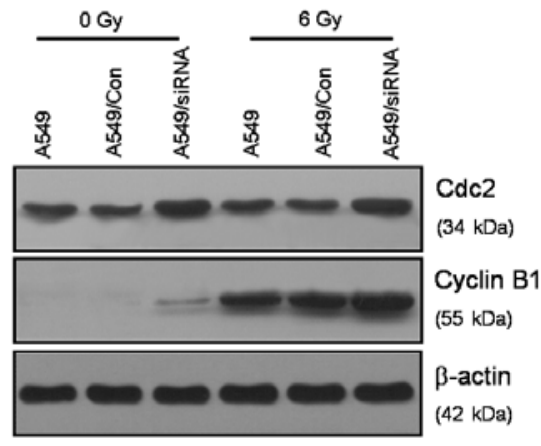

Figure 3. TOB1 regulates cell cycle distribution of lung cancer cells after irradiation. (A) TOB1-transfected NCI-H1975 cells and (B) TOB1-siRNA-transfected A549 cells were irradiated with 6 Gy X-rays. Cell cycle distribution was determined $24 \mathrm{~h}$ later by flow cytometric analysis. (C and D) Western blot analysis was performed to determine the expression of proteins associated with cell cycle progression. All the experiments were performed independently at least three times.
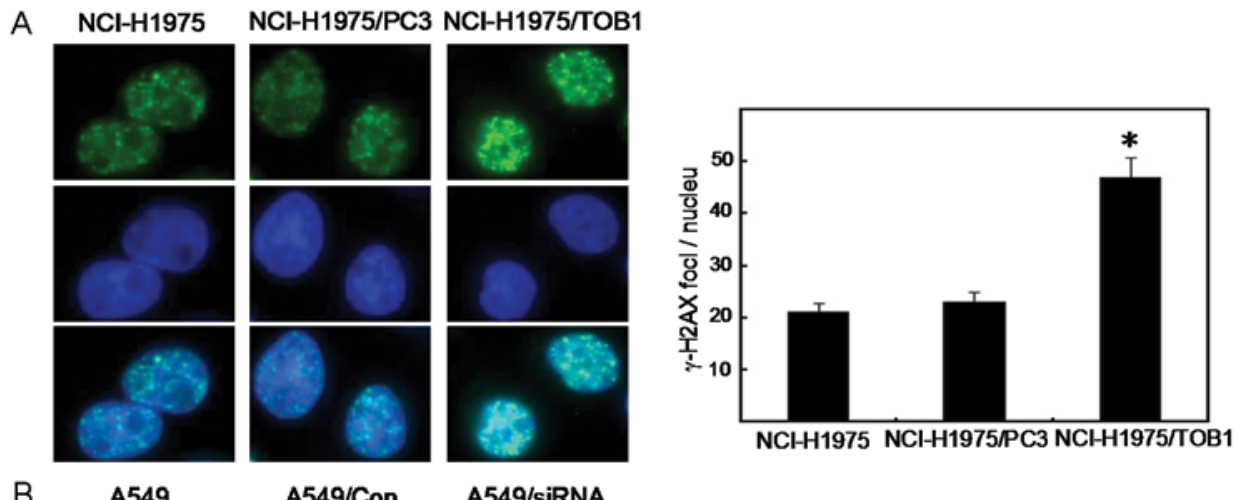

B
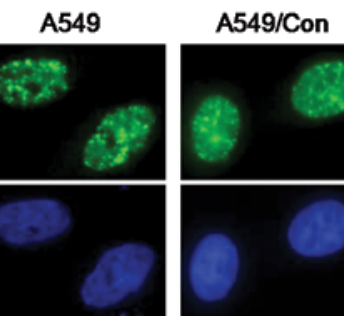

A549/siRNA
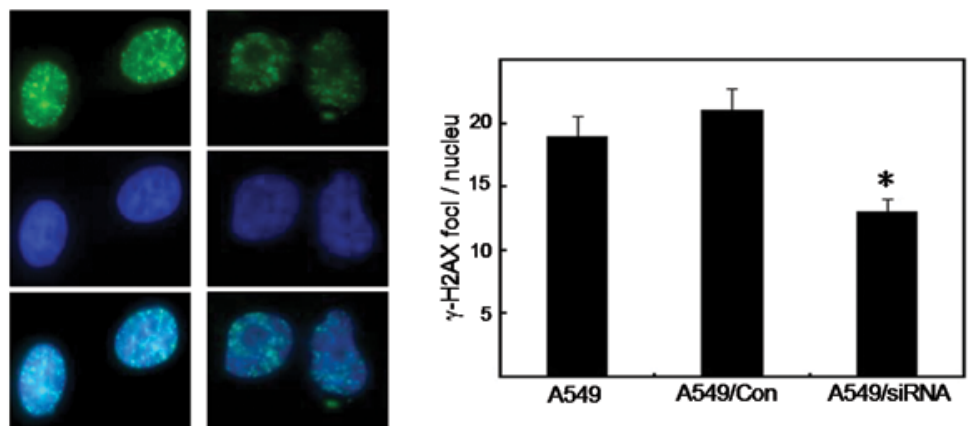

Figure 4. TOB1 regulates $\gamma$-H2AX focus formation after irradiation. (A) TOB1-transfected NCI-H1975 cells and (B) TOB1-siRNA-transfected A549 cells were irradiated with a single dose of $6 \mathrm{~Gy}$ X-rays followed by immunofluorescence $1 \mathrm{~h}$ later. Unirradiated cells were used as control cells. Quantitative analysis of $\gamma$-H2AX foci per cell averaged over 50 cells per data point. All the experiments were performed independently at least three times. ${ }^{*} \mathrm{P}<0.05$ vs. the control group. 
A

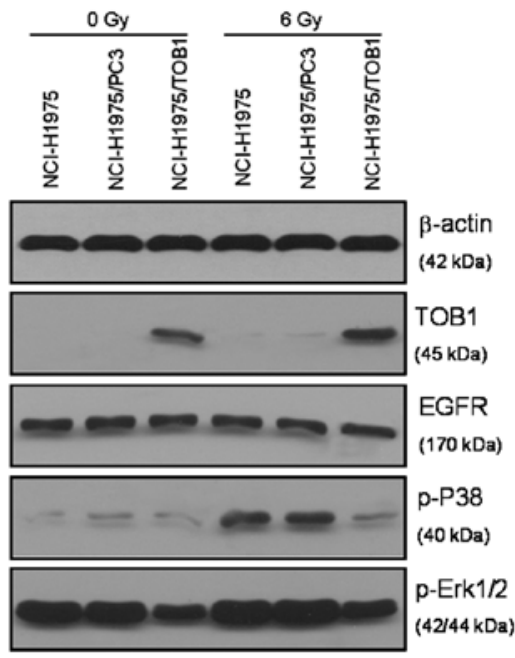

B

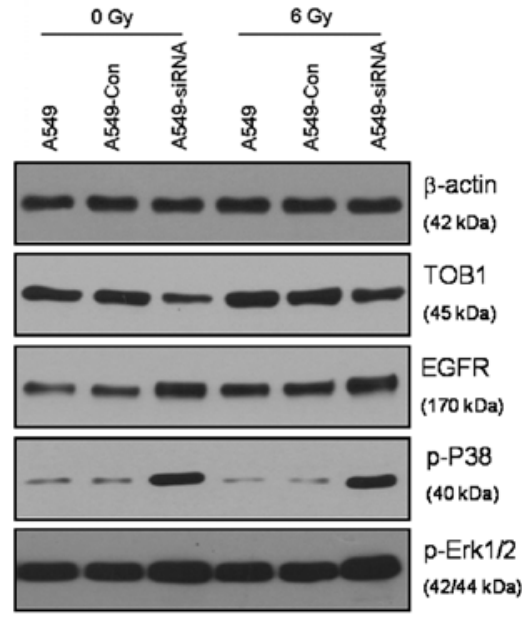

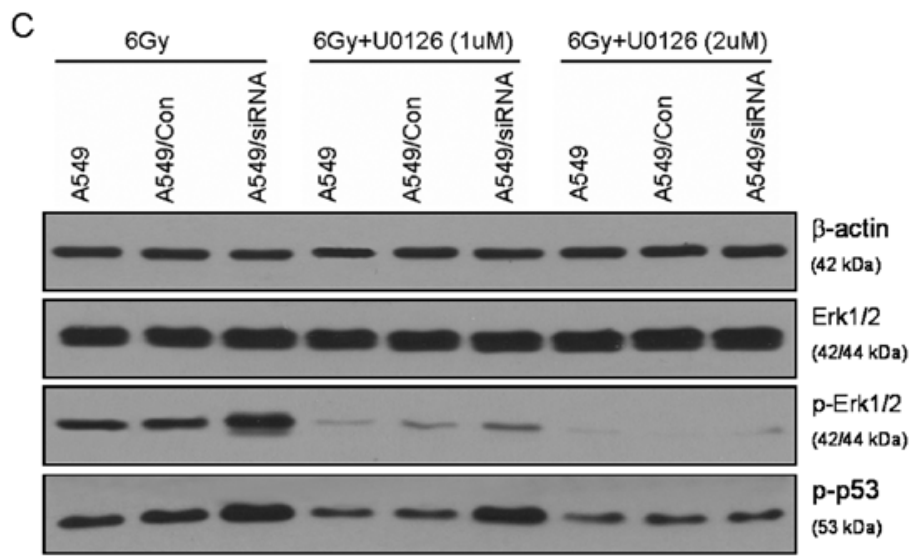

Figure 5. TOB1 modulates the phosphorylation of p53 via the MAPK/ERK signaling pathway after irradiation. Western blot analyses were performed to determine the expression of EGFR and the downstream cascades in (A) TOB1-overexpressing NCI-H1975 cells and (B) TOB1-siRNA-transfected A549 cells. Cells were exposed to $6 \mathrm{~Gy}$ of irradiation, and $50 \mu \mathrm{g}$ of proteins was resolved using SDS-PAGE, followed by immunoblotting with the indicated specific antibodies of the MAPK signaling pathway. (C) One hour after irradiation treatment, TOB1-siRNA-transfected A549 cells were cultured in the absence or presence of the MAPK/ERK inhibitor (UO126). The expression level of phospho-p53 (p-p53) was determined by western blot analysis. Blots are representative of three experiments.

cells in the G2/M phase was increased compared with radiation alone $(\mathrm{t}=6.32, \mathrm{P}<0.05)$ (Fig. 3B). The expression levels of several important proteins associated with the cell cycle were analyzed to investigate the significant cell cycle changes in TOB1-transfected NCI-H1975 cells. Results revealed that cyclin B1 was significantly suppressed (Fig. 3C). A549 cells knocked down for TOB1 exhibited opposite effects for the regulation of the cell cycle-associated proteins (Fig. 3D).

TOB1 regulates $\gamma$-H2AX focus formation in lung cancer cells following irradiation. The induction of DNA-DSBs analyzed by the formation of $\gamma-\mathrm{H} 2 \mathrm{AX}$ foci was measured $1 \mathrm{~h}$ after irradiation of lung cancer cells, non-transfected or transfected with TOB1 recombinant plasmid or TOB1-siRNA. This procedure aimed to identify the molecular mechanisms of radiosensitization of TOB1 and investigate its effects on the initial DNA damage response to IR radiation. Results showed that the radiation-induced $\gamma$-H2AX focus formation was significantly increased in TOB1-transfected NCI-H1975 cells 2 h postradiation compared with the control cells $(\mathrm{t}=11.20, \mathrm{P}<0.05)$ (Fig. 4A), suggesting inhibition of DNA repair. TOB1 knock- down demonstrated opposite effects in the siRNA-transfected A549 cells ( $\mathrm{t}=7.12, \mathrm{P}<0.05)$ (Fig. 4B).

TOB1 is involved in serine 15 phosphorylation of p53 via the mitogen-activated protein kinase (MAPK)/ERK signaling pathway after irradiation. Western blot analysis was performed to identify the targets of TOB1 that are involved in the enhancement of radiosensitivity. EGFR, which is frequently involved in the pathogenesis of human epithelial tumors, adversely affects prognosis and treatment outcome due to EGFR-mediated therapy resistance (7). The results of this study revealed that TOB1 overexpression did not significantly inhibit EGFR expression in the EGFR-mutated NCI-H1975 cells. However, TOB1 knockdown enhanced EGFR expression in the siRNA-tranfected A549 cells. In EGFR downstream effectors, TOB1 augmented a significantly suppressed radiation-induced phosphorylation of ERK1/2 and p38, while it had no obvious effects on the expression of these proteins. Phosphorylation and subsequent activation of $\mathrm{p} 53$, as well as its transcriptional induction, are key initial responses to cell cycle distribution and DNA damage (8). The specific 
signaling cascade involved in this response was investigated in the present study using mitogen-activated or extracellular signal-regulated protein kinases 1 and 2 (MEK1/2) inhibitor (U0126) specific to the MAPK/ERK pathway. The increased phospho-p53 in A549 cells knocked down for TOB1 was inhibited by MEK1/2 inhibitor (Fig. 5C), suggesting that TOB1 mediates the phosphorylation of p53 at serine 15 by modulating the MAPK/ERK pathway.

\section{Discussion}

More than $70 \%$ of cancer patients currently receive radiotherapy during their treatment course (9-11). However, conquering the intrinsic radioresistance and increasing the precision of dose delivery to target tumor remain difficult for radiotherapy (12). To the best of our knowledge, the results of the present study indicated for the first time that irradiation stimulated TOB1 expression in the lung cancer cell lines A549 and NCI-H1975. A significant increase in the TOB1 protein level was observed when a dose of $\geq 2$ Gy was used. The time-dependent induction of TOB1 was increased $6 \mathrm{~h}$ after radiation. Thus, TOB1 is potentially involved in the regulation of radiosensitivity in lung cancer cells.

In the present study, we investigated whether the combination of the regulation of TOB1 expression and IR radiation affects the kinetics of cell death compared with cells treated with radiation alone. Using the clonogenic survival assay, we found that TOB1 overexpression reduces the clonogenic growth of NCI-H1975 cells after irradiation compared with the parental and mock-treated cells. On the contrary, TOB1 knockdown protects A549 cells from radiation when compared with the control cells.

Radiation induces a complex cellular response, which activates and coordinates cell cycle checkpoints and damages repair (13). Most mammalian cells exhibit transient delays in the $\mathrm{G} 1$ and $\mathrm{G} 2$ phases after irradiation allowing the cell to correct possible defects (6). In the present study it was shown that TOB1 overexpression decreases the radiationinduced NCI-H1975 cell accumulation in the G2 phase. TOB1 knockdown demonstrated opposite effects in A549 cells, suggesting that TOB1 regulates the cell cycle redistribution of lung cancer cells after radiation. We analyzed the expression levels of several cell cycle-associated proteins and found that the expression levels of cyclin $\mathrm{B} 1$ and $\mathrm{Cdc} 2$ were significantly suppressed in TOB1-transfected NCI-H1975 cells. TOB1 knockdown demonstrated opposite effects in siRNA-transfected A549 cells.

Phosphorylation of H2AX plays an important role in the recruitment of repair- and damage-signalling factors to the site of DNA breaks (14-16). $\gamma$-H2AX is widely used in monitoring the extent of DSB induction and analyzing the effectiveness of novel biological therapies (17). TOB1 produced a higher amount of $\gamma$-H2AX foci in TOB1-transfected NCI-H1975 cells after irradiation. Radiation-induced $\gamma-\mathrm{H} 2 \mathrm{AX}$ was significantly inhibited in A549 cells knocked down for TOB1. Based on these results, it is suggested that the phenotypes of increased radiosensitivity may thus reflect distinct and differentially regulated factors of the DNA damage response.

Extensive evidence indicates that EGFR signaling plays an important role in mediating the radiosensitivity and activation of multiple signaling pathways (18). Principle data suggest that EGFR heterodimer or homodimer formation induced by ligand binding activates the intracellular tyrosine kinase domain, induces additional downstream pathways and affects radiosensitivity (19). Inhibition of the MAPK pathway may allow inhibition of signaling by multiple upstream receptors and intermediates, such as EGFR $(20,21)$. Our results revealed that TOB1 overexpression did not inhibit EGFR expression in EGFR-mutated NCI-H1975 cells, while TOB1 knockdown enhanced EGFR expression in siRNA-transfected A549 cells. Regarding the EGFR downstream signaling pathway, we examined the expression of the phosphorylation of ERK1/2 and $\mathrm{p} 38$, which is mainly associated with radiosensitivity. Results revealed that TOB1 overexpression significantly inhibited the activation of MAPK, while TOB1 suppression enhanced MAPK activation. This suggests that TOB1 affects the phosphorylation of MAPK via an EGFR-independent pathway.

The MAPK pathway directly affects the phosphorylation of p53 at multiple sites, which are key initial responses to cell cycle distribution and DNA damage (22-24). Our results revealed ed that TOB1 knockdown strongly enhanced radiation-induced phosphorylation of p53 in A549 cells. This result suggests that these cells possess a more robust DNA damage surveillance and possibly a repair mechanism that helps them either adapt to or overcome critical radiation-induced DNA damage. The inhibitor specific to MAPK/ERK (U0126) was used following radiation. The ability of the MEK $1 / 2$ inhibitor to affect the serine 15 phosphorylation of $\mathrm{p} 53$ indicates the direct role of MAPK/ERK in modulating this particular cellular radiation response in siRNA-transfected A549 cells, while gefitinib did not affect the specific event (data not shown).

In conclusion, this study suggests that TOB1 may be a novel molecular target of irradiation. TOB1 was shown to modulate the radiosensitivity of lung cancer cells through the MAPK/ERK signaling pathway via an EGFR-independent pathway. Gene therapeutic approaches may enhance the radiotherapeutic benefit and overcome the resistance of EGFR inhibitors in lung cancer therapy by increasing TOB1 expression.

\section{Acknowledgements}

This study was supported by grants from the Doctoral Fund of the Ministry of Education of China (grant no. 20103201120016), the College Nature Science Foundation of Jiangsu Province (grant no. SZ126821), the Social Development Projects of Kunshan City (grant no. KS1224) and the Priority Academic Program Development of Jiangsu Higher Education Institutions (PAPD).

\section{References}

1. Jemal A, Bray F, Center MM, et al: Global cancer statistics. CA Cancer J Clin 61: 69-90, 2011.

2. Matsuda S, Kawamura-Tsuzuku J, Ohsugi M, et al: Tob, a novel protein that interacts with p185erbB2, is associated with anti-proliferative activity. Oncogene 12: 705-713, 1996.

3. Jia S and Meng A: Tob genes in development and homeostasis. Dev Dyn 236: 913-921, 2007.

4. Jiao Y, Ge CM, Meng QH, et al: Adenovirus-mediated expression of Tob1 sensitizes breast cancer cells to ionizing radiation. Acta Pharmacol Sin 28: 1628-1636, 2007. 
5. Jiao Y, Sun KK, Zhao L, et al: Suppression of human lung cancer cell proliferation and metastasis in vitro by the transducer of ErbB-2.1 (TOB1). Acta Pharmacol Sin 33: 250-260, 2012.

6. Ahsan A, Hiniker SM, Davis MA, et al: Role of cell cycle in epidermal growth factor receptor inhibitor-mediated radiosensitization. Cancer Res 69: 5108-5114, 2009.

7. Wheeler DL, Dunn EF and Harari PM: Understanding resistance to EGFR inhibitors - impact on future treatment strategies. Nat Rev Clin Oncol 7: 493-507, 2010.

8. Shieh SY, Ikeda M, Taya Y, et al: DNA damage-induced phosphorylation of p53 alleviates inhibition by MDM2. Cell 91: 325-334, 1997.

9. Yang P: Epidemiology of lung cancer prognosis: quantity and quality of life. Methods Mol Biol 471: 469-486, 2009.

10. Molina JR, Yang P, Cassivi SD, et al: Non-small cell lung cancer: epidemiology, risk factors, treatment, and survivorship. Mayo Clin Proc 83: 584-594, 2008.

11. Ahmed KM and Li JJ: NF-kappa B-mediated adaptive resistance to ionizing radiation. Free Radic Biol Med 44: 1-13, 2008.

12. Gerber DE: EGFR inhibition in the treatment of non-small cell lung cancer. Drug Dev Res 69: 359-372, 2008.

13. Meyn RE, Munshi A, Haymach JV, et al: Receptor signaling as a regulatory mechanism of DNA repair. Radiother Oncol 92 316-322, 2009

14. Mahaney BL, Meek K and Lees-Miller SP: Repair of ionizing radiation-induced DNA double-strand breaks by non-homologous end-joining. Biochem J 417: 639-650, 2009.

15. Morgan MA, Parsels LA, Maybaum J, et al: Improving gemcitabine-mediated radiosensitization using molecularly targeted therapy: a review. Clin Cancer Res 14: 6744-6750, 2008 .
16. Chastel C, Jiricny J and Jaussi R: Activation of stress-responsive promoters by ionizing radiation for deployment in targeted gene therapy. DNA Repair (Amst) 3: 201-215, 2004.

17. He HT, Fokas E, You A, et al: Siah1 proteins enhance radiosensitivity of human breast cancer cells. BMC Cancer 10: 403, 2010.

18. Toulany M, Kasten-Pisula U, Brammer I, et al: Blockage of epidermal growth factor receptor-phosphatidylinositol 3-kinaseAKT signaling increases radiosensitivity of K-RAS mutated human tumor cells in vitro by affecting DNA repair. Clin Cancer Res 12: 4119-4126, 2006.

19. Chung EJ, Brown AP, Asano H, et al: In vitro and in vivo radiosensitization with AZD6244 (ARRY-142886), an inhibitor of mitogen-activated protein kinase/extracellular signal-regulated kinase 1/2 kinase. Clin Cancer Res 15: 3050-3057, 2009.

20. Li MW, Mruk DD and Cheng CY: Mitogen-activated protein kinases in male reproductive function. Trends Mol Med 15: 159-168, 2009.

21. Kim Y, Coppey M, Grossman R, et al: MAPK substrate competition integrates patterning signals in the Drosophila embryo. Curr Biol 20: 446-451, 2010.

22. Arany PR, Flanders KC, DeGraff W, et al: Absence of Smad3 confers radioprotection through modulation of ERK-MAPK in primary dermal fibroblasts. J Dermatol Sci 48: 35-42, 2007.

23. She QB, Chen N and Dong Z: ERKs and p38 kinase phosphorylate $\mathrm{p} 53$ protein at serine 15 in response to $\mathrm{UV}$ radiation. $\mathrm{J}$ Biol Chem 275: 20444-20449, 2000.

24. Dumaz N and Meek DW: Serine15 phosphorylation stimulates p53 transactivation but does not directly influence interaction with HDM2. EMBO J 18: 7002-7010, 1999. 